\title{
10
}

\section{High-speed free-space laser communication}

\author{
Yoshinori Arimoto and Norihisa Hiromoto \\ Communications Research Laboratory, MPT \\ 4-2-1 Nukui-Kita, Koganei, Tokyo 184 JAPAN \\ Tel: +81-423-27-7512, Fax: +81-423-27-6699, \\ E-mail:arimoto@crl.go.jp
}

\begin{abstract}
The laser communication system proposed here for short-distance horizontal ground links and for the link between a ground station and a geostationary satellite will provide eye-safe and power-efficient communication by using a wavelength between 1.5 and $2.2 \mu \mathrm{m}$. This proposal is based on the results of a space laser communication system study, and the proposed system will use high-speed optical devices and adaptive optics that actively compensate wavefront distortions. A link budget study shows that a system with a 10 -Gbps bit-rate between a geostationary satellite and a ground station can be constructed using technology already available. The power efficiency of the system is expected to be improved by using a multilevel pulse position modulation scheme, short-pulse lasers, and fiber amplifiers.
\end{abstract}

\section{Keywords}

Free-space laser communication, geostationary satellite, adaptive optics, fiber amplifier 


\section{INTRODUCTION}

The high speed and large bandwidth offered by lightwave communications makes them a very attractive means of meeting the future demand for personal multimedia communication including text, voice, still-picture, and video information. The high-speed fiber-optic communication system has been growing rapidly recently, and time division multiplexing (TDM) and wave division multiplexing (WDM) technologies will provide the basis for its continued growth. The bit-rate in trunk lines is expected to soon be between $100 \mathrm{Gbps}$ and 1 Tbps.

In the case of the free-space laser communication, however, the bit-rate of the link for short range in-door use, such as IrDA link, is currently limited to sereral mega bit per second, and the bit-rate of short-range LAN extenders has been extended to hundreds mega bit per second using large aperture optics and sharp laser beams. The bandwidth limitation due to eye safety will not be relaxed further because the maximum permissible exposure (MPE) for near-infrared lasers is so low that it will be very difficult to reach bit-rates above $1 \mathrm{Gbps}$. Longer wavelengths, such as $1.5 \mu \mathrm{m}$ or $2.0 \mu \mathrm{m}$, should therefore be used because the MPE for that wavelength region is less stringent than that for the near infrared.

For intersatellite laser communication links there is another bandwidth limitation. The link bit-rate is considered to be power-limited rather than dispersion-limited as in the case of fiber optic links because the range between geostationary satellites is from $40000 \mathrm{~km}$ to $70000 \mathrm{~km}$ and the free-space propagation loss due to the beam divergence is as much as $300 \mathrm{dBs}$. In this case, very narrow laser beams should be used with large aperture optics, and this would require an accurate pointing and tracking system. A high power laser source, such as one with an optical power of $1 \mathrm{~W}$ and with diffraction-limited beam quality, and a sensitive photodetector whose sensitivity is nearly quantum limited are also important in space laser communications. Combining these space laser communications technologies and the recent advances in longer-wvavelength fiber optic communications, we will be able to achieve good power efficiency and eyesafety in the free-space laser communication systems.

This paper will describe (a) the results of laser communications experiments using the Engineering Test Satellite VI (ETS-VI) and (b) the concept of the optical feeder-link system, which connects the geostationary satellite and a ground station, based on the high-speed optical devices developed for ground-based fiber-optic communications and on the adaptive optics that uses real-time feedback control to compensate wavefront distortions due to atmospheric turbulences. This paper also describe (c) a multilevel pulse position modulation system with error-correction coding that should provide extremely good power efficiency. 


\section{SATELLITE LASER COMMUNICATION EXPERIMENT USING ETS-VI}

The first experiment on laser communication between a geostationary-altitude orbiting satellite and a ground station was performed by the Communications Research Laboratory (CRL) using the ETS-VI which was launched into highelliptical orbit on 28 August $199+$ and completed its operations on 9 July 1996 (Araki, et. al. 1997). The experiment was performed when the satellite was visible from CRL's ground station and the satellite attitude could be maintained for the onboard laser transmitter to point to the ground station.

A laser diode oscillating at $0.83 \mu \mathrm{m}$ with the peak output power of $30 \mathrm{~mW}$ was used for down-link transmission, and an argon ion laser oscillating at $0.5145 \mu \mathrm{m}$ with the continuous output power of $10 \mathrm{~W}$ was used for up-link transmission. The main optical ground station was at the CRL, in a suburb of Tokyo. From November 1995 to May 1996 the CRL was engaged in an international cooperative experiment with the Jet Propulsion Laboratory (JPL) of the USA, taking advantage of such favorable site conditions as the fewer atmospheric effects above JPL's Table Mountain Facility (TMF) ground station 2300 meters above sea level. This joint project provided us with a detailed evaluation and analysis of the link budget and onboard equipment. Figure 1 shows the configuration of this cooperative experiment.

The experiment showed that the up-link laser transmission is susceptible to serere

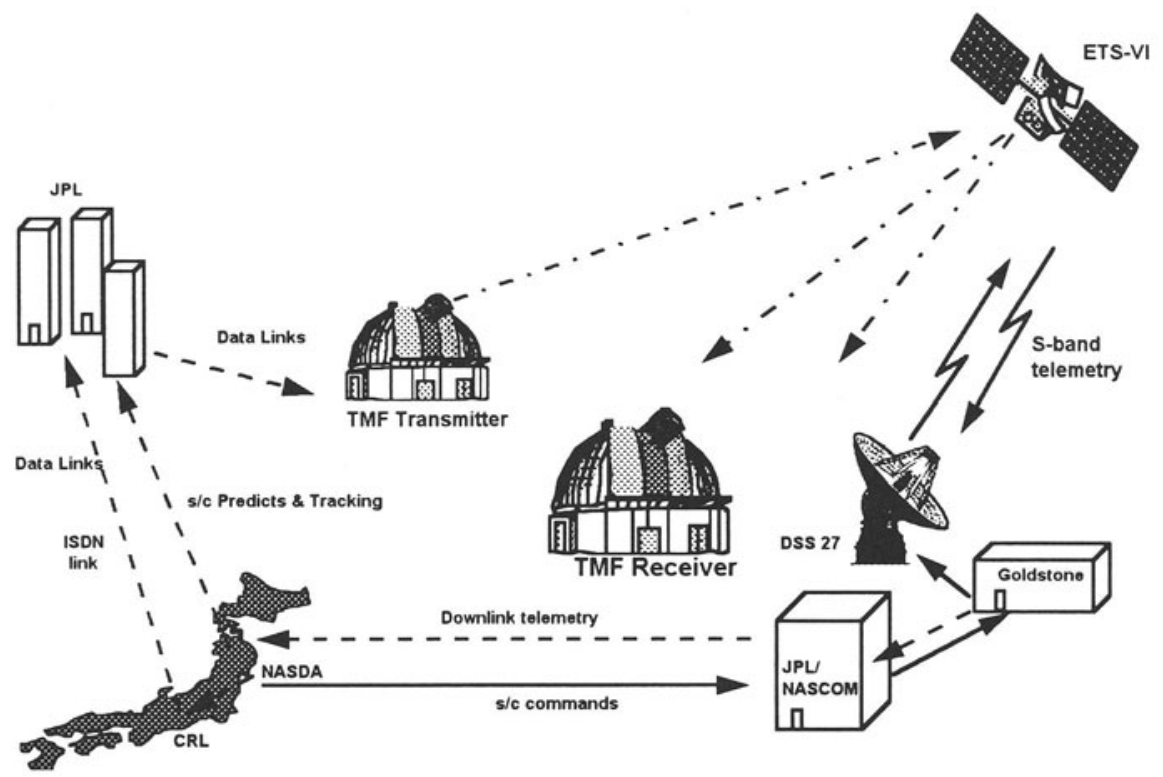

Figure 1 Configuration of the satellite laser link experiment using the ETS-VI in collaboration with NASA/JPL (Wilson and Lesh, 1997). 


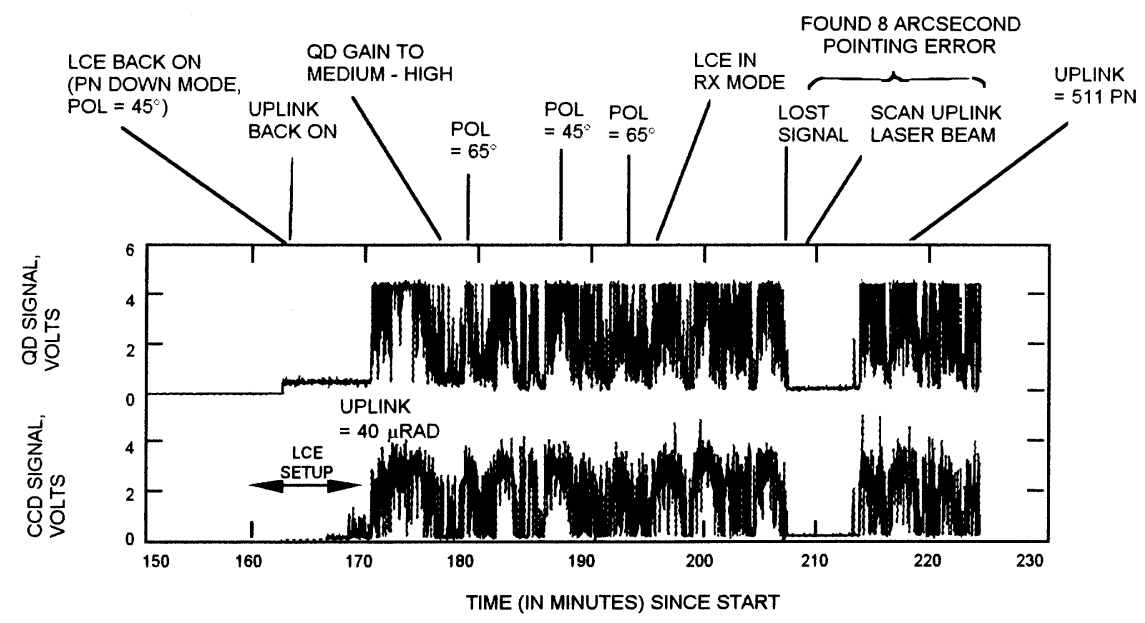

Figure 2 Uplink laser transmission from the TMF ground station (Lesh, 1997).

attenuation and scintillation caused by atmospheric turbulence (Arimoto, et. al. 1995). Figure 2 shows a typical experimental sequence of uplink transmission from the ground station at the Table Mountain Facility of JPL. A PN sequence with $1.02+\mathrm{MHz}$ Manchester coding was transmitted from the ground station to the satellite and the bit error rate (BER) was monitored at the satellite. In the downlink experiment performed at the Table Mountain ground station, the telemetry data in the laser communication equipment onboard the ETS-VI was transmitted from the satellite to the ground station via a $1.024-\mathrm{Mbps}$ optical link, and the results show that the BER remained better than $10^{-4}$ even during several minutes when the link condition was good (Jeganathan and Toyoshima, 1997). Figure 3 shows an example of the downlink bit-error measurement results.

To improve the up-link transmission quality during atmospheric turbulence, the CRL has recently been considering the application of adaptive optics (AO) like those used in large astronomical telescopes (Roddier, Northcott, and Graves, 1991). The down-link laser light transmitted from the satellite can be thought of as an artificial guide star in the AO system, which will be able to compensate the severe signal attenuation and scintillation due to wavefront distortion. The use of adaptive optics will require that several problems be solved, such as the increase of the isoplanatic angle in order to cover the point-ahead angle of the laser transmission, chromatic aberrations due to the difference between the wavelengths of the transmitting and receiving lasers, and the increase of effective wind-speed due to fast satellite movement. Adaptive optics, however, will be a key technology for the future ground-to-space laser transmission.

The CRL program developing AO technology for space laser communication was started in April 1996. Its objective is to provide a 10-Gbps optical feeder-link between a geostationary satellite and several ground stations within 10 years. The 


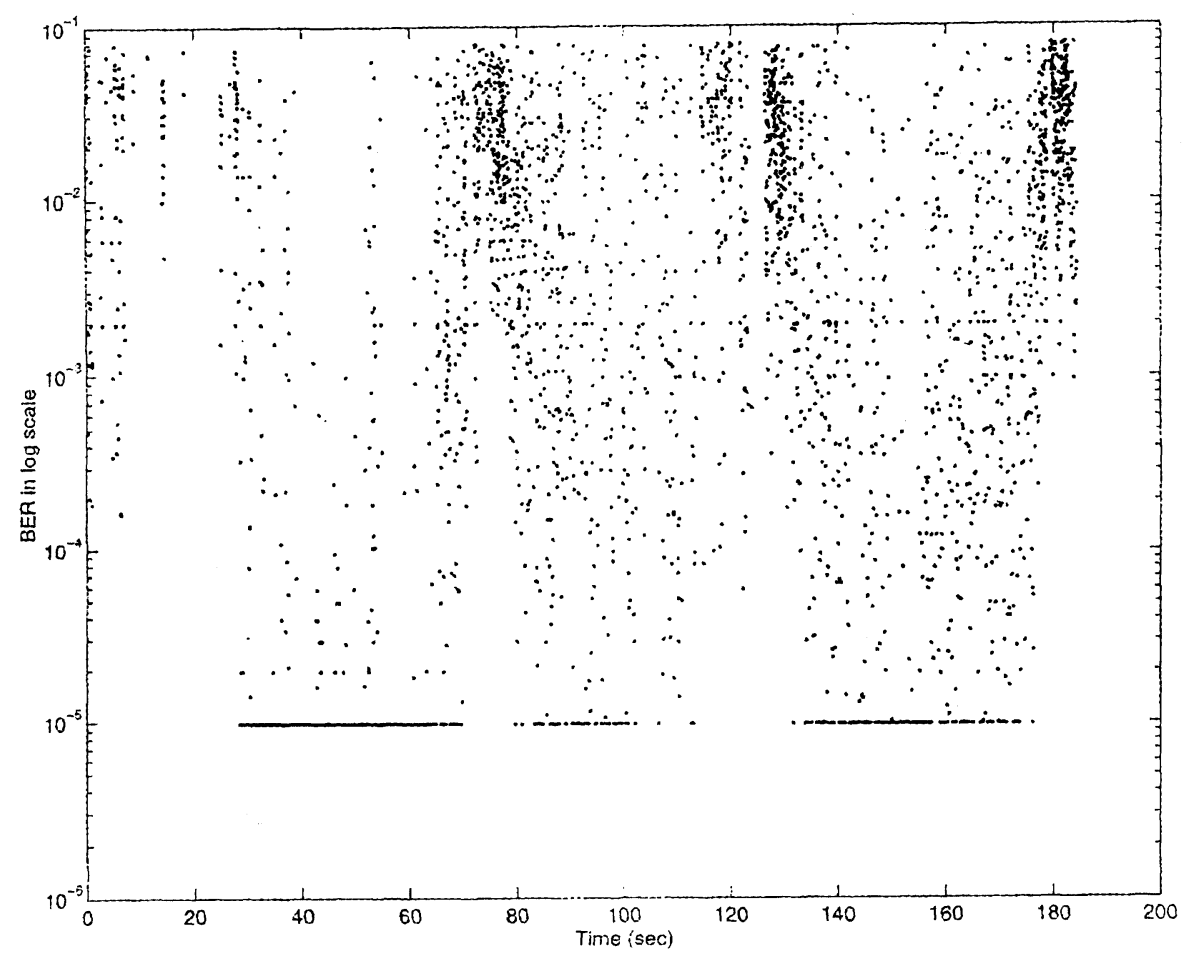

Figure 3 Bit error rate measured in a three-minute-long PN data sequence on 27 April 1996. Each point represents 0.1 seconds of data transmitted at 1.024 Mbps (Wilson and Lesh, 1997).

first on-site atmospheric turbulence measurements in this program were made, using a wavefront sensor for the adaptive optic system and a 1.5-m-telescope receiver system, in May of 1995 and in February and August of 1996. We also estimated the turbulence's coherence length and temporal characteristics and are currently designing an experimental demonstrator of the AO system for the 10Gbps optical feeder-link.

\section{STUDY ON THT OPTICAL FEEDER-LINK SYSTEM}

\subsection{System concept}

Optical feeder-links are attractive for use in satellite communication systems because they can provide data rates on the order of $10 \mathrm{Gbps}$ without any mutual interference (Arimoto, 1997). The microwave feeder-link systems, even in the millimeter wave region, cannot provide such high data rates and it is very difficult to use the microwave frequency bands efficiently without any interference. The 


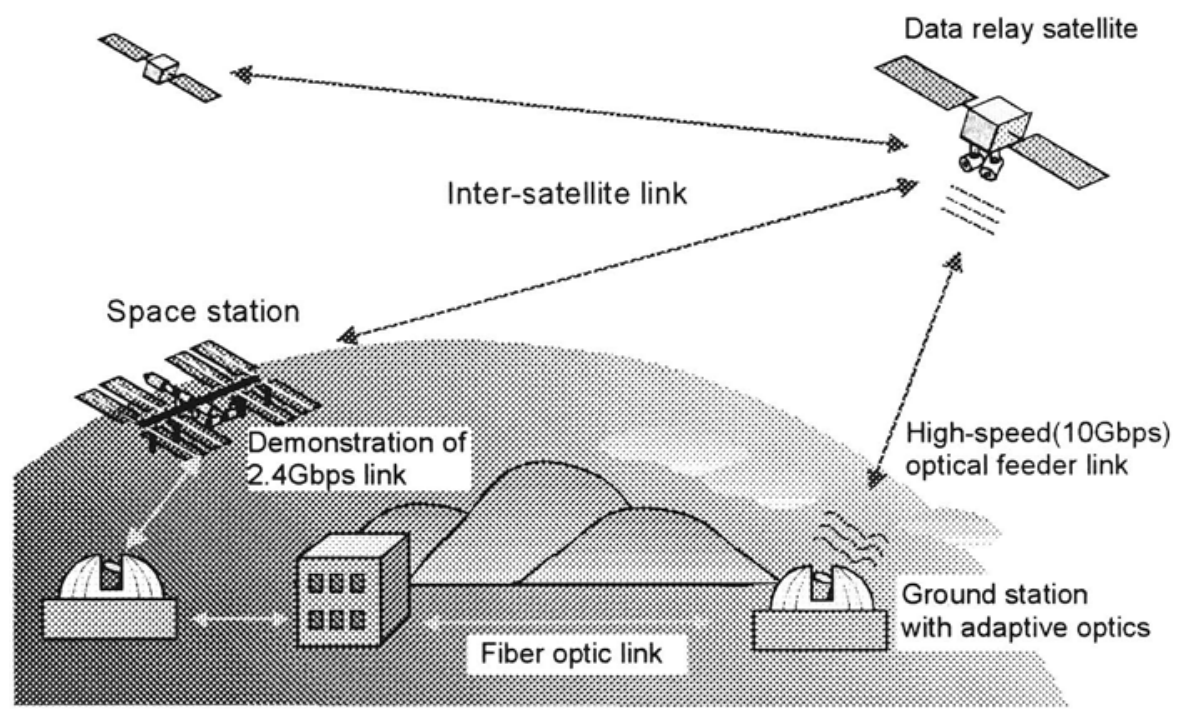

Figure 4 System concept of the optical feeder-link.

availability of ground-to-satellite optical links, however, is limited because the link performance is strongly affected by weather conditions, such as clouds and fog. We therefore focused on two areas critical to the efficient use of optical feeder-links:

- communication of a large amount of data in a short time (burst mode communication), and

- a high-bit-rate interconnection or gateway link between the terrestrial and space networks.

A two-minute optical link with a bit-rate of $2.4 \mathrm{Gbps}$, for example, can transfer an amount of data comparable to the amount on seven digital video discs. Such a throughput would be enough for a data relay system that does not require quick responses or interactive communication.

Figure 4 shows the concept of the optical feeder-link system connecting the space network with the terrestrial network. The optical inter-satellite link is useful because there is nothing in space to disturb the propagation of the laser beam. In the feeder-link between the ground station and the relay satellite, however, near the aperture of the ground telescope there is strong atmospheric turbulence a heights up to $5 \mathrm{~km}$, and this turbulence interferes with the up-link laser transmission. The rest of the propagation path is free of turbulence, so it is possible to compensate the atmospheric turbulence by using an adaptive-optics (AO) system in the ground station.

Besides, it is not difficult to install optical fiber links to several ground stations with good site conditions and to connect them with the terrestrial network. Figure 


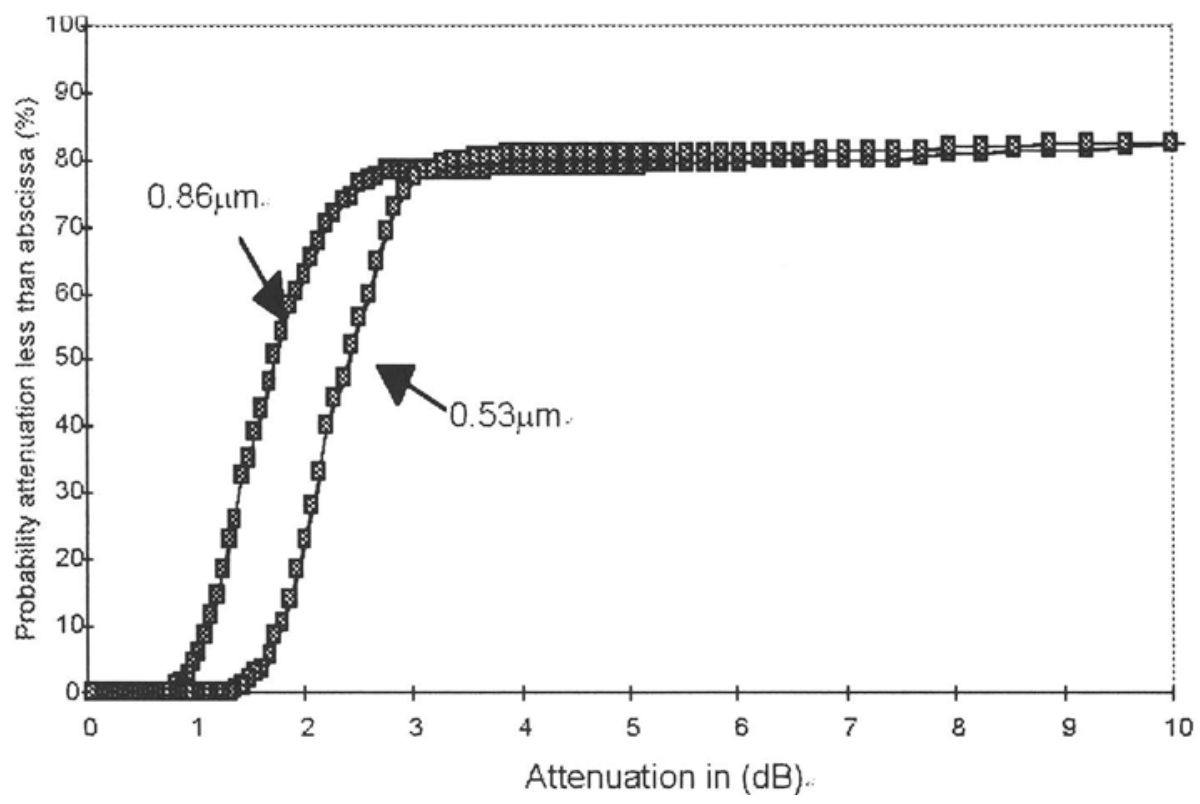

Figure 5 Cumulative probability distributions for the TMF observatory: 860-nm and 532-nm filters (Lesh, 1997).

5 shows typical visibility statistics at the Table Mountain Observatory of the Jet Propulsion Laboratory (Lesh, 1997).

\subsection{Link budget calculation}

A link budget for this optical feeder-link system was calculated, assuming stateof-the-art devices in 1996, and table 1 shows the results of this calculation. For both the high-power transmitter and the low-noise receiver, we chose $1.5-\mu \mathrm{m}$ technologies and assumed the differential PSK-encoding/differential detection system based on the experimental result obtained by the MIT Lincoln Laboratory (Livas, Swanson, Chinn, and Kintzer, 1995). These combinations were reported to show near shot-noise limited sensitivity which is the ultimate performance in receiver sensitivity.

In the case of a 1.5- $\mu \mathrm{m}$ wavelength, an isoplanatic angle, which limits the field of view of the compensation in adaptive optics, is greater than the point-ahead angle for the satellite in geostationary orbit if the elevation angle to the satellite is large enough not to pass the thick turbulent layer. Using low-order adaptive-optics compensation, we could thus expect an effective gain of about $10 \mathrm{~dB}$, which corresponds to a reduction of fluctuation by the same amount. One component important to achieving this gain is the fiber coupling device inserting the light gathered by the telescope at the ground station into the single-mode Er-doped fiber 
Table 1. Link budget for the 10-Gbps optical feeder-link system.

\begin{tabular}{|c|c|c|}
\hline & $\begin{array}{l}\text { Downlink } \\
\text { (satellite } \rightarrow \text { ground) }\end{array}$ & $\begin{array}{l}\text { Uplink } \\
\text { (ground } \rightarrow \text { satellite) }\end{array}$ \\
\hline Wavelength & $1.55 \mu \mathrm{m}$ & $1.55 \mu \mathrm{m}$ \\
\hline Laser power & $1 \mathrm{~W}$ & $2 \mathrm{~W}$ \\
\hline Transmitting antenna $^{(a)}$ & $20 \mathrm{~cm}$ & $100 \mathrm{~cm}$ \\
\hline Antenna gain & $109.15 \mathrm{~dB}$ & $123.13 \mathrm{~dB}$ \\
\hline Distance & $38,000 \mathrm{~km}$ & $38,000 \mathrm{~km}$ \\
\hline Free-space loss & $-289.77 \mathrm{~dB}$ & $-289.77 \mathrm{~dB}$ \\
\hline Receiving antenna ${ }^{(a)}$ & $100 \mathrm{~cm}$ & $20 \mathrm{~cm}$ \\
\hline Antenna gain & $123.13 \mathrm{~dB}$ & $109.15 \mathrm{~dB}$ \\
\hline Atmospheric loss, etc. ${ }^{(b)}$ & $-10.1 \mathrm{~dB}$ & $-9.6 \mathrm{~dB}$ \\
\hline Receiving power & $-37.59 \mathrm{dBm}$ & $-34.09 \mathrm{dBm}$ \\
\hline Sensitivity & 70 photons/bit & 70 photons/bit \\
\hline Required power & $-40.47 \mathrm{dBm}$ & $-40.47 \mathrm{dBm}$ \\
\hline Margin & $2.9 \mathrm{~dB}$ & $6.4 \mathrm{~dB}$ \\
\hline
\end{tabular}

(a) Antenna efficiency is assumed to be $50 \%$.

(b) Absorption loss $=3.0 \mathrm{~dB}$, Strehl ratio due to atmospheric turbulence $=0.27$, coupling loss for wavefront sensing $=0.5 \mathrm{~dB}$.

amplifier. We also assumed that the atmospheric absorption is less than $3.0 \mathrm{~dB}$, since there are no strong atmospheric absorption bands in the $1.5-\mu \mathrm{m}$ wavelength region.

Consequently, high-speed optical feeder-links will enable us to use an alloptical space communication network. Even if the site diversity provided by several ground stations will be required, the high data rate will justify the cost of constructing the ground stations and the installing the optical fiber link.

\subsection{Design of the transceiver}

The key component in the transceiver design for the 10-Gbps feeder-link system is the Er-doped fiber amplifier, and in 1996 we started trial fabrications of two different types of systems using the same fiber amplifier. One was the DPSK coherent system based on the MIT report showing that the optical power required for a BER of $10^{-6}$ is 50 photons/bit, and the other was an IM/DD system using return-zero (RZ) pulses. Figure 6 shows the configuration of the DPSK coherent type transceiver using fiber amplifiers.

The sensitivity of the IM/DD system is theoretically $3.0 \mathrm{~dB}$ less than that of the DPSK coherent system. If we take into account the fiber amplifier characteristics of the output-power saturation effect, however, we found that the IM/DD system using short RZ pulse modulation will provide better power-efficiency than will a conventional IM/DD system using the NRZ pulses. This is because the ON-duty 
Table 2 Major parameters of the feeder-link transceiver.

\begin{tabular}{|c|c|}
\hline \multicolumn{2}{|c|}{ High-power fiber amplifier } \\
\hline Output power: & $400 \mathrm{~mW}$ \\
\hline Pumping power: & $\begin{array}{l}1200 \mathrm{~mW}, 2 \text {-wave division and 2-polarization } \\
\text { division multi-plexing }\end{array}$ \\
\hline $\begin{array}{l}\text { Pump wavelength: } \\
\text { Polarization: }\end{array}$ & $\begin{array}{l}1.48 \mu \mathrm{m} \\
\text { maintained }\end{array}$ \\
\hline \multicolumn{2}{|c|}{ Low-noise fiber amplifier } \\
\hline Wavelength: & $1.552 \mu \mathrm{m}$ \\
\hline Noise Figure: & less than $5.0 \mathrm{~dB}$ \\
\hline Output power: & 8.5-17.5 dBm (selectable by 3-bit jumper switch) \\
\hline Small signal gain: & more than $36.0 \mathrm{~dB}$ \\
\hline Pump wavelength: & $0.98 \mu \mathrm{m}$ (first stage), $1.48 \mu \mathrm{m}$ (second stage) \\
\hline Polarization: & maintained \\
\hline \multicolumn{2}{|l|}{ Receiver } \\
\hline Bit-rate & $10 \mathrm{Gbps}$ \\
\hline Sensitivity: & 90 Photons/Bit at $10^{-9} \mathrm{BER}$ \\
\hline \multicolumn{2}{|l|}{ Coarse tracking system } \\
\hline Detector: & $\begin{array}{l}\text { InGaAs photodiode array integrated with a CMOS } \\
\text { readout multiplexer (SU128-1.7) }\end{array}$ \\
\hline Array layout: & $128 \times 128$ pixels \\
\hline Quantum efficiency: & more than $80 \%$ at $1.55 \mu \mathrm{m}$ \\
\hline
\end{tabular}

ratio in RZ pulsing is smaller than the one in NRZ pulsing.

We confirmed experimentally that nearly shot-noise-limited performance, such as a sensitivity of 90 photons/bit at a BER of $10^{-9}$, can already be achieved using currently available low-noise fiber amplifiers and a $10 \mathrm{Gbps}$ RZ-type receiver.

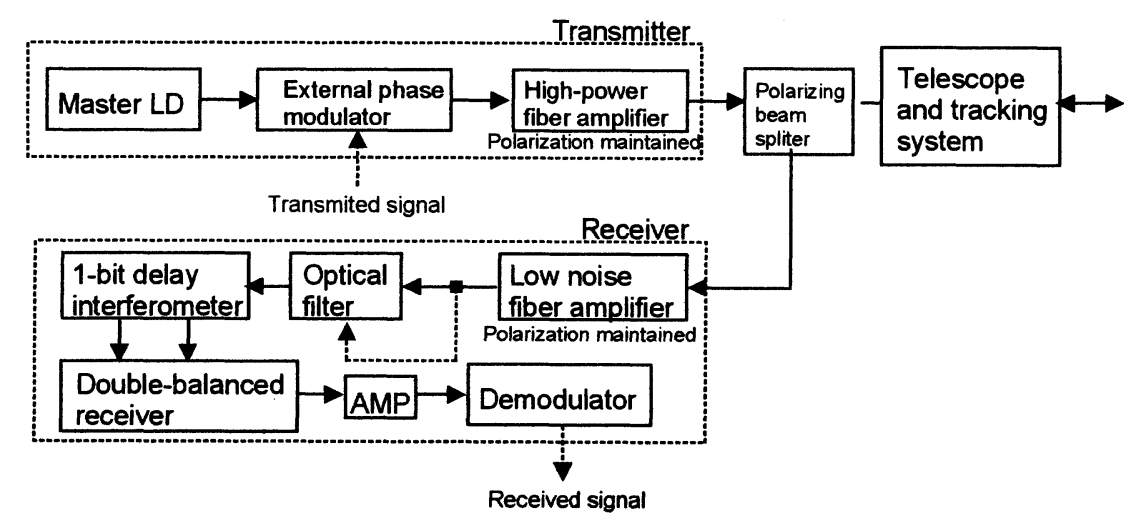

Figure 6 Configuration of the feeder-link transceiver using fiber amplifiers. 
The optical feeder-link system requires that the polarization of the incident laser light be maintained in the fiber amplifier. Because the waveguide directional coupler used in the PSK differential detection receiver is polarization dependent, we chose to use polarization-maintaining fiber amplifiers for both the high-power amplifier and the low-noise amplifier. The major parameters in the current design of the feeder link transceiver are summarized in Table 2.

The Er-doped fiber amplifier is used to achieve a high power output, as large as $400 \mathrm{~mW}$ with a near-Gaussian beam profile, in the laser communication transmitter and good sensitivity, with nearly shot-noise-limited performance, in the laser communication receiver. The space segment of the system is similar to that used for communication between a geostationary satellite and a low earthorbiting satellite.

\subsection{Design of the adaptive-optics system}

It is very important for the optical feeder-link system to guide the downlink laser light gathered by the telescope into the single-mode fiber in the coherent detection system or the system which uses a fiber amplifier. For this purpose there are special fiber-coupling devices such as a mechanical nutation fiber coupler, but, these devices are incapable of correcting the wavefront error due to the atmospheric turbulence. Adaptive optics seems to be the best technology for concentrating the incident laser light into a single-mode fiber amplifier because the general purpose of adaptive optics is to transform uncorrected incident light into a sharp spot image, e.g., on a CCD image sensor (Kaufmann, 1995).

In our optical feeder-link system the compensation of the atmospheric

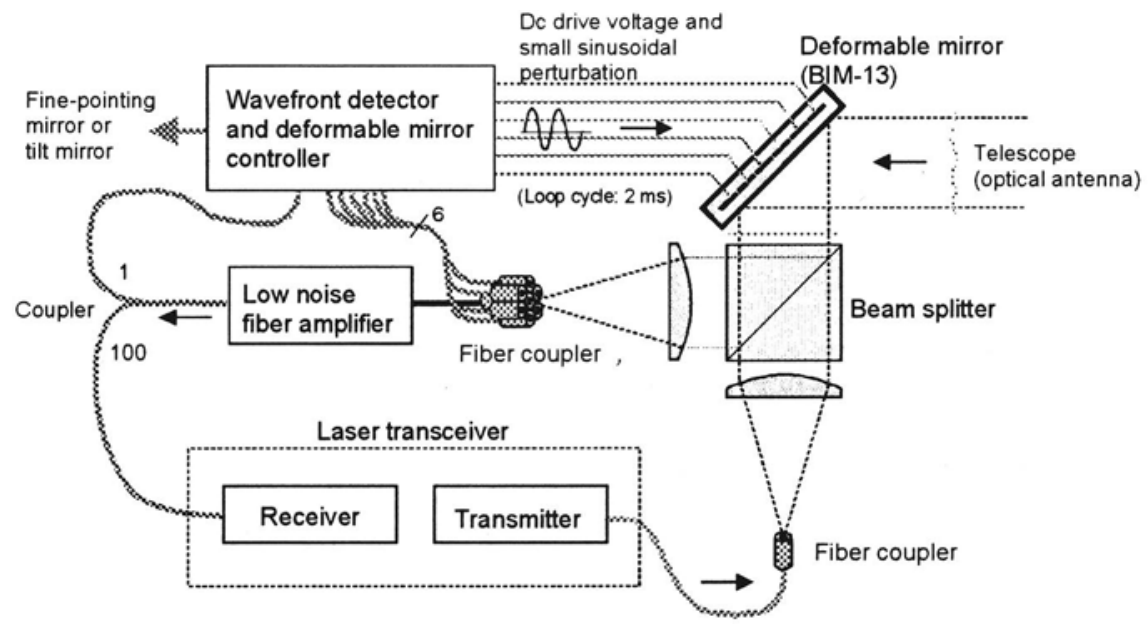

Figure 7 Configuration of the adaptive-optics fiber coupler. 
wavefront error in the uplink propagation path is essential for the efficient use of the large aperture of the ground telescope. Compensation in the downlink path is also important for high efficiency in the single-mode fiber coupler. We therefore designed an adaptive-optics fiber coupler for the optical feeder-link system.

As shown in Figure 7, the coupler consists of a 13-electrode bimorph mirror (Gaffard, Jagourel, and Gigan, 1994) and a wavefront sensor using a bundle of fiber couplers, a low-noise fiber amplifier, and wavefront reconstructor (detector). The central port of the bundle fiber coupler is also used as the receiver's input port. The remaining six ports are used to detect the low-order wavefront errors. such as tilt, defocus, and astigmatism. To select and separate each of the wavefront error modes, a small sinusoidal perturbation is applied to the voltage driving the bimorph mirror. Our design has following features:

- tilt tracking and higher-order wavefront error detection,

- automatic coalignment between the tilt tracking system and the receiver,

- minimum receiver sensitivity degradation due to coupling loss.

InGaAs pin photodiodes will be used in the wavefront detector. We are fabricating prototypes of the fiber coupler and the wavefront detector to confirm their feasibility. We are also considering the possibility of using the liquid crystal device instead of the bimorph mirror (Klaus, Ide. Morokawa. Hayano. and Arimoto) if the requirement of the control bandwidth of the adaptive-optics system is less stringent than that of the current design.

Some of the technology for the ground segment will be verified in a ground-10satellite experiment using the Optical Interorbit Communication Engineering Test Satellite (OICETS) that is being developed by National Space Development Agency of Japan (NASDA) and that will be launched into a low earth orbit in the year 2000. The CRL will take part in this experiment using the main optical ground station that was used for the ETS-VI experiments. We have been authorized to perform a laser communication demonstration experiment using the Japanese Experimental Module (JEM) on the International Space Station. This experiment will demonstrate 2.5 -Gbps down-links between the JEM and several ground stations.

\section{SHORT-RANGE FREE-SPACE LASER COMMUNICATION SYSTEM}

Infrared free-space (wireless) LAN systems with bit-rates of about $10 \mathrm{Mbps}$ for inoffice use and a bit-rate of $155 \mathrm{Mbps}$ for between-buildings use have been developed. These systems operate at wavelengths of $0.7-0.8 \mu \mathrm{m}$ and they use a GaAs/AlGaAs LED or laser diode as a light source and use a Si-PIN photodiode or APD (avalanche photodiode) as a detector. 


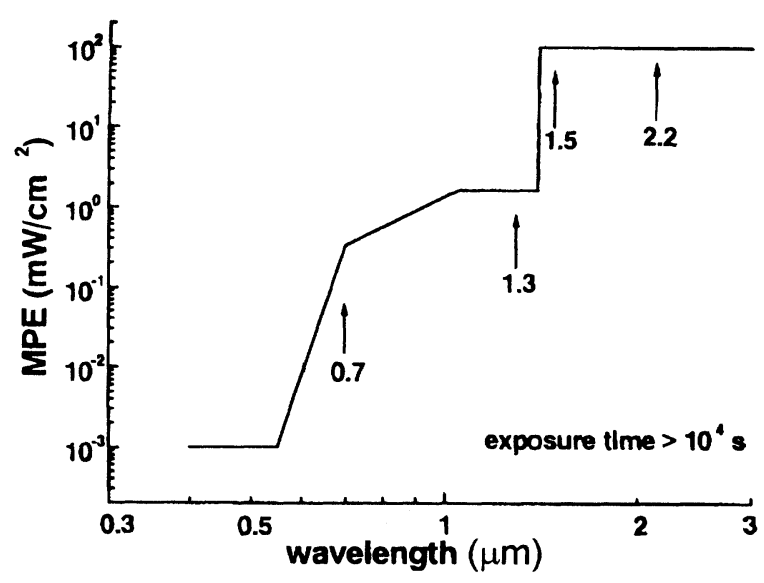

Figure 8 Laser power density of the maximum permissible exposure (MPE) as a function of the wavelength.

For higher data rates and for communication over longer distances in the ground-based free-space communications links, however, for eye-safety it will be necessary to use wavelength longer than $1.4 \mu \mathrm{m}$, because such systems will require light sources that are much more powerful. The laser power density of the maximum permissible exposure (MPE) to which the human eye can be exposed for more than $10^{4}$ seconds is shown in Fig. 8 as a function of wavelength. The MPE at $0.7 \mu \mathrm{m}$ is only $0.3 \mathrm{~mW} / \mathrm{cm}^{2}$ but at wavelengths longer than $1.4 \mu \mathrm{m}$ is $100 \mathrm{~mW} / \mathrm{cm}^{2}$

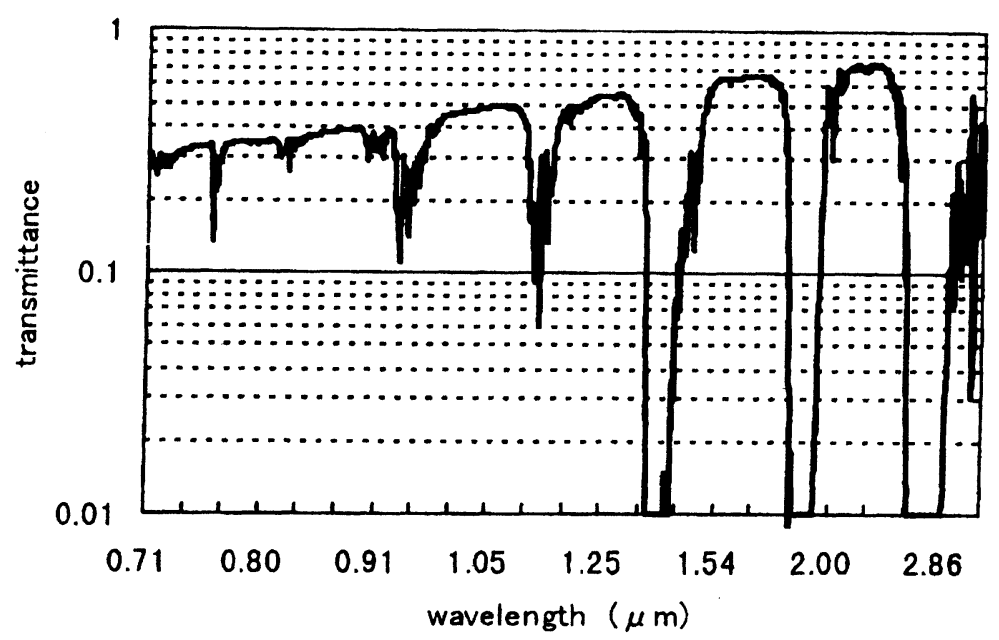

Figure 9 Transmittance through $2 \mathrm{~km}$ of atmosphere. 


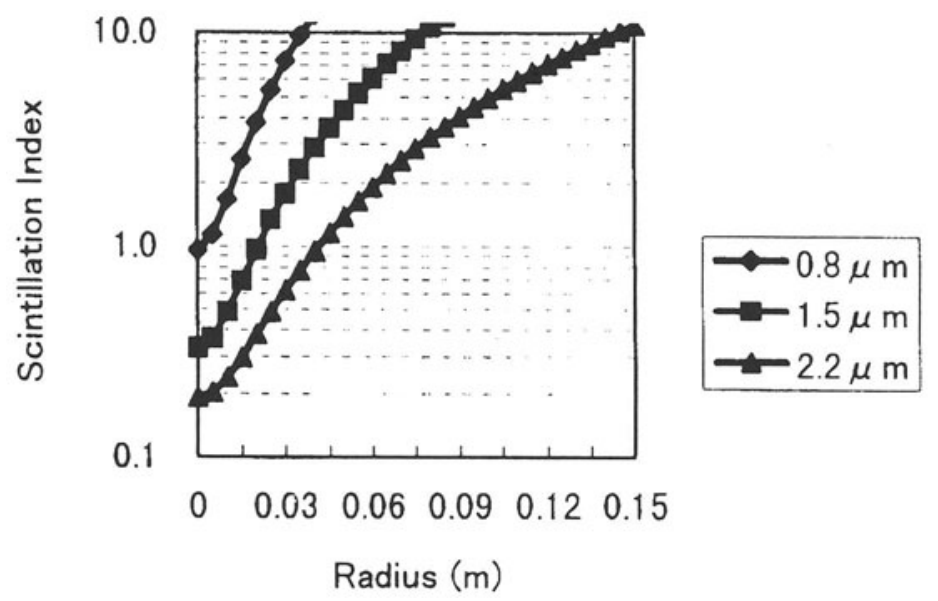

Figure 10 Scintillation index profile for a propagation length of $2 \mathrm{~km}$ in the atmospheric turbulence $C_{n}^{2}=1.7 \times 10^{-14} \mathrm{~m}^{-2 / 3}$.

(JIS C 6802-1991).

The wavelength used for long distance horizontal-path free-space communication links should be chosen such that (1) the extinction (i.e., absorption and scattering) due to atmospheric molecules and aerosols is small. (2) the background levels are low, and (3) the scintillation caused by atmospheric turbulence is small.

The transmittance of light over a 2-km distance in a standard atmosphere (U. S. Standard Atmosphere, 1976, horizontal path, altitude of $0 \mathrm{~m}$, temperature of 15 degrees Celsius, humidity of $46 \%$, and pressure of $1013.25 \mathrm{hPa}$ ) is shown in Figure 9 as a function of wavelength. The maximum transmittance is for wavelengths near $2.2 \mu \mathrm{m}$. The transmittance plotted in Figure 9 was calculated using the MODTRAN atmospheric model (ONCORE,1993). The daytime atmospheric radiance calculated by this software was lowest around $2.5 \mu \mathrm{m}$, in the valley between the scattered sunlight and the thermal emission of the atmosphere.

Calculated scintillation index profiles for wavelengths of $0.8,1.5$, and $2.2 \mu \mathrm{m}$ are shown in Figure 10. Each profile is for a Gaussian beam of $2.5-\mathrm{cm}$ radius transmitted through $2 \mathrm{~km}$ of atmosphere with a turbulence $C_{n}{ }^{2}$ of $1.7 \times 10^{-14} \mathrm{~m}^{-2 / 3}$. The formulation for a Gaussian beam propagating in a turbulent atmosphere (Andrews, Phillips, and Yu, 1995) was used in calculating these profiles, and Figure 10 shows that a small enough scintillation index is obtained only for a $2-\mu \mathrm{m}$ wavelength.

The discussion above indicates that better propagation characteristics for highrate long distance links will be provided by a communication system using a longer wavelength (e.g., $2.2 \mu \mathrm{m}$ in Figure 10). A laser communication system operating in this wavelength region, however, will have to await the development of a low- 
Table 3 Example of the link budget for a short-range 1.5- $\mu \mathrm{m}$ laser communication system.

\begin{aligned} & Wavelength $1.5 \mu \mathrm{m} \\ &$ Transmitting laser power $1 \mathrm{~mW} \\ &$ Transmitting optics loss $-5.23 \mathrm{~dB} \\ &$ Transmitteing beam radius $2 \mathrm{~cm} \\ &$ Power density at the transmitter $1.53 \mathrm{~mW} / \mathrm{cm}^{2} \\ &$ Propagation length $2000 \mathrm{~m} \\ &$ Atmospheric transmittance 0.63 \\ & Effective beam radius at receiver $21.8 \mathrm{~cm} \\ &$ Required pointing accuracy $3.61 \mathrm{arcsec} \\ &$ Receiver aperture diameter $10 \mathrm{~cm} \\ &$ Beam expansion loss $-6.76 \mathrm{~dB} \\ &$ Receiver optics loss $-5.23 \mathrm{~dB} \\ &$ Receiving power $-19.24 \mathrm{~dB} \\ &$ Receiver sensitivity $90 \mathrm{photons} / \mathrm{bit} \\ &$ Data rate $10 \mathrm{Gbps} \\ &$ Required optical power $-39.24 \mathrm{~dB} \\ &$ Link margin $20.00 \mathrm{~dB} \\ &$\hline\end{aligned}

noise. high-speed detector and a laser that is both power efficient and reliable. We are therefore dereloping $\mathrm{In}_{1-\mathrm{x}} \mathrm{Ga}_{\mathrm{x}} \mathrm{As}$-PIN photodiodes with a $2.4-\mu \mathrm{m}$ cutoff wavelength $(\mathrm{x}=0.26)$, a $1.3-\mathrm{GHz}$ bandwidth, and a sensor diameter greater than $100 \mu \mathrm{m}$.

In the $1.5-\mu \mathrm{m}$ wavelength region, however, many optical communication devices have already been developed. The most important is the optical amplifier using an Er-doped single mode fiber.

Table 3 is an example of the link budget for a short distance laser communication link using the $1.5 \mu \mathrm{m}$ technology described in the Section 3. A 10-Gbps communication link over a 2-km horizontal path is feasible even with a 1-mW optical transmitter if the Er-doped fiber amplifier is used in the front end of the receiver. The optical power efficiency can be expected to be further improved by using a recently developed very-short-pulse laser source and a multilevel pulse position modulation scheme (Lesh, 1982). The proposed system nonetheless has several draw-backs: one is the accurate tracking/pointing required, which will increase the system cost, and another is the possibility of burst errors due to tracking errors and scintillation in the propagation path. The ATM network, one of the most promising high-speed network protocols, will require that the links are largely free of burst errors. The large link margin listed in Table 3 should be devoted to reducing the burst error if the free-space laser link is to be used in the ATM network. Another way avoid the effects of burst errors is by using strong error correction code. 


\section{CONCLUSION}

The proposed high-speed free-space laser communication system is suitable for use in short-distance horizontal ground links and in the link between a ground station and a geostationary satellite. The technologies which have been developed for space laser communication can be used to achieve good power efficiency by using near-quantum-limited sensitive receivers, narrow beam laser transmission and precise tracking/pointing system. Selection of the wavelength for the laser communication is also important. We have shown that longer wavelengths such as those between $1.5 \mu \mathrm{m}$ and $2.0 \mu \mathrm{m}$, will be attractive because their eye safety standards are less stringent and they are less sensitive to the effects of atmospheric turbulence.

The proposed system is based on (a) the technology used in and the experience gained in the space laser communication demonstration using the ETS-VI, (b) high-speed optical devices developed for ground-based high-speed fiber-optic communications, and (c) adaptive optics that uses real-time feedback control to compensate wavefront distortions due to atmospheric turbulence.

A link budget study indicates that a system with a 10-Gbps bit-rate between the geostationary satellite and the ground station can be put together using current state-of-the-art devices and Er-doped fiber amplifiers. The recent developments in short-pulse lasers, fiber amplifiers, and a multilevel pulse position modulation system with error-correction coding have made it possible to construct a free-space laser communication system with extremely good power efficiency.

\section{REFERENCES}

Araki, K., Toyoshima, M., Takahashi, T., Fukazawa, T., Toyoda, M., Shikatani, M. and Arimoto, Y. (1997) Experimental operations of laser communication equipment onboard ETS-VI satellite. SPIE, 2990-29.

Arimoto, Y., Toyoshima, M., Toyoda, M., Takahashi, T., Shikatani, M. and Araki, K. (1995) Preliminary result on laser communication experiment using ETSVI. SPIE, 2381.

Jeganathan, M. and Toyoshima, M. (1997) Data analysis results from the GOLD laser communications. SPIE, 2990-08.

Wilson, K. E., Jeganathan, M., James, J., Xu, G. and Lesh, J. R. (1996) Results from Phase-1 and Phase-2 GOLD experiments. JPL TDA Progress Report 42-127.

Roddier, F., Northcott, M. and Graves, J. (1991) A Simple low-order adaptive optics system for near-infrared applications. Publication of the Astronomical Society of the Pacific, 103, 131-149. 
Arimoto, Y., Hayano, Y. and Klaus, W. (1997) High speed optical feeler-link system using adaptive optics. SPIE, 2990-15.

Lesh. J. R. (1997) Overview of the NASA/JPL lasercom program. CRL International Topical IVorkshop on Space Laser Communication, 7-13.

J. Livas, J., Swanson, E.. Chinn, S. and Kintzer. E. (1995) High data rate sysstems for space applications. SPIE, 2381.

Kaufmann. J. (1995) Performance limits of high-rate space-to-ground optical communications through the turbulent atmospheric channel. SPIE, 2381.

Gaffard, J., Jagourel, P. and Gigan, P. (1994) Adaptive optics: Description of available components at LASERDOT. SPIE Adaptive Optics in Astronomy; 2201.

Klaus, W., Ide, M., Morokawa, S., Hayano, Y. and Arimoto, Y. (1997) Efficient liquid crystal wavefront modulator. SPIE, 3015-11.

JIS C 6802-1991 (1991) Radiation safety standards for laser products.: IEC 8251984, Radiation safety of laser products, equipment classification, requirements, and user's quide.

U. S. Standard Atmosphere (National Oceanic and Atmospheric Administration, National Aeronautics and Space Administration, and United States Air Force, Washington, D. C., 1976) NOAA-S/T76-1562.

ONCORE, Personal Computer Version of the MODTRAN 2 Atmospheric Model (ONTAR Corporation, Massachusetts, 1993) Version 2.0.

Andrews, L. C., Phillips. R. L. and Yu. P. T. (1995) Optical scintillations and fade statistics for a satellite-communications system. Applied Optics, 34, 77+27751.

Lesh, J.R. (1982) Optical communications research to demonstrate 2.5 bits/detected photon. IEEE Communications Magazine, 35-37.

\section{Biography}

Yoshinori Arimoto was born in Wakayama, Japan on July 3, 1954. He received the M.S. degree in physics from Osaka University, Japan in 1979. He has been with the Communication Research Laboratory (CRL), Ministry of Posts and Telecommunications from 1979. From 1983 to 1985 he temporarily moved out to Telecommunications Satellite Corporation of Japan (TSCJ), where he engaged in the orbit planning of the broadcasting satellite and communications satellite. From 1990 to 1993 he joined into ATR Optical and Radio Communications Research Laboratory, where he engaged in the research of inter-satellite laser communication system. Now, he heads the space technology section at Space Communications Division of CRL and his interest in research is communicationrelated space infrastructure, such as free-space laser communication, tele-science, space debris issues, etc. 